FINAL REPORT

\title{
A Robust Miniature Multi-Function, Dense WDM Demultiplexer/receiver
}

Supported by the Air Force Office of Scientific Research

Under the contract No. F49620-98-C-0066

Submitted by Contractor:

Eagle Research \& Development, $L L C$

370 Interlocken Blvd., $4^{\text {th }} \mathrm{fl}$.

Broomfield, CO 80021

(303) 474-1826

Principal Investigator:

Co Principal Investigator:

Eagle Optoelectronics, LLC \#
Dr. Heinz Willebrand

Dr. Jon Sauer

EOI/F.03

"The views and conclusions contained in this document are those of the author's and should not be interpreted as necessarily representing the official policies, either expressed or implied, of the Government." 
Fulic reporting burden for this coliection of information is estimated to average 1 hour pes response. including th

gathering and maintaining the data needed, and completing and reveiwing the collection of information. Send $c$

Davis Highway Suite 1204 A Aling suggestions for reducing this burden, to Washington Headquarters Services, \begin{tabular}{l|l|l} 
1. AGENCY USE ONLY ILeave Blank; & 2. REPCRT DATE
\end{tabular}

4. TITLE AND SUETITLE

June 28,2000

Final Report 15/08/98 - 14/02/99

A Robust Miniature Multi-Function, Dense WDM Demultiplexer/receiver

6. AUTHOR(S)

Dr. Heinz Willebrand

Dr. Jon Sauer

7. PERFORMING ORGANIZATION NAME(S) AND ADDRESSIES

Eagle Optoelectronics, LLC

370 Interlocken Blvd., $4^{\text {th }} \mathrm{fl}$.

Broomfield, CO 80021

5. FUNDING NUMBERS

H16103

$1660 / 01$

9. SPONSORING/MONITORING AGENCY NAME(S) ANO ADDRESSIES)

Air Force Office of Scientific Research

801 N. Randolph St.

Arlington, VA 22203-1977

8. PERFORMING ORGANIZATION

REPORT NUMBEF

EO1/F.03

11. SUPPLEMENTARY NOTES

12a. DISTRIBUTION/AVAILABILITY STATEMEN

Approved for public release,

distribution unlimited

13. ABSTRACT (Maximum The broad goal was to advance the state-of-the-art in wDM receiver

technology based on microcavity resonators. In order to do this,

with such tools, demonstrated 2D capable of handling 3D, complex geometries.

fabricate could be replatated $2 \mathrm{D}$ designs that are very difficult to

place all the precision features as acturable 3D designs. These 3D designs

to fabricate verticas as epitaxial layers that are much easier

To advance the goal of manufact horizontal features at the same precision.

- fabricated some prototype

Iimited success

(which the CalTech design code to run on the San Diego Tera supercomputer

inserted the recently-derable I/O system development)

finite-difference algorithm intomory- and cycle-saving cole non-standard feasible

which would implemting animated visualizations based on an open source packagle

on generic UNIX/Linux visualization of the core computations accessible

Contion platforms

14. SUBJECT TERMS "user-friendy"

"user-friendly" modules usable by other groups.

\begin{tabular}{|c|c|c|c|}
\hline & & & 16. PRICE CODE \\
\hline $\begin{array}{l}\text { 17. SECURITY CLASSIFICTION OF } \\
\text { REPORT } \\
\text { UNCLASSIFIED }\end{array}$ & $\begin{array}{l}\text { 18. SECURITY CLASSIFICATION OF } \\
\text { THIS PAGE } \\
\text { UNCLASSIFIED }\end{array}$ & $\begin{array}{l}\text { 19. SECUAITY CLASSIFICATION OF } \\
\text { ABSTRACT } \\
\text { UNCLASSIFIE D }\end{array}$ & 20. LIMITATION OF ABSTRACT \\
\hline
\end{tabular}

Standard form 298 (Rev. 2-89)

Prescribed by ANSI Std. 239-1 


\section{Table of Contents}

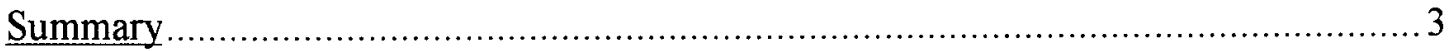

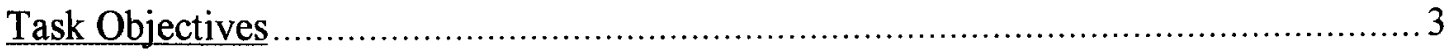

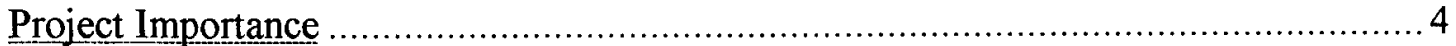

Project Background and General Methodology ............................................. 5

Project State after Completion of Phase 1 ..................................................... 12

Intellectual Property, Marketing Efforts and Business/Commercialization Strategy ... 12

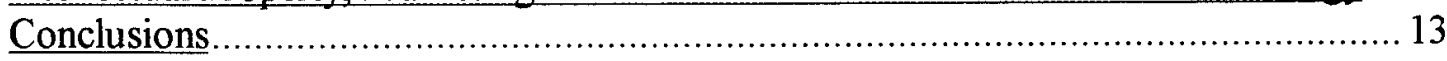

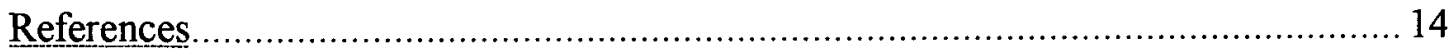




\section{Summary}

This is the final report for the Phase I SBIR project " $A$ Robust Miniature MultiFunction, Dense WDM Demultiplexer/receiver." The work described in this report was performed by Eagle Research and Development, LLC, formerly Eagle Optoelectronics, LLC, between December, 1998 and October, 1999. The following tasks were completed at the end of the Phase I effort:

- Acquired finite difference numerical method [1] from James Cole, Naval Research Laboratories, in Fortran source code. Began porting algorithms in C source to Tera Supercomputer in San Diego Supercomputing Center.

- Opened channels of communication with Professor Axel Scherer of the California Institute of Technology. Intradepartmental finite difference modeling tools given to us as a possible template to modify using James Cole's algorithm.

\section{Task Objectives}

This project attempted to provide a new computer simulation and visualization tool to aid in the physical design of the WDM microcavity filter. We have collected literature describing the theoretical and experimental nature of microcavity resonators used as optical filters. A review of the literature shows that the initial microring resonators, based on weakly guiding material, had a free-spectral range (FSR) of less than the ITU standard of $100 \mathrm{GHz}$ channel spacing, thereby not really behaving as filters. Vernier effect using double and triple filters has been described as a method to increase the FSR. However, the FSR for the weakly guided rings is still not large enough to accommodate many channels. We intend to use computers to simulate the effects of the 
electromagnetic fields on a structure of known geometry and composition. The ability to model these effects could greatly aid in determining the best geometry and composition of the microcavity and minimize the number of iterations in the physical fabrication process. Reducing the number of fabrication cycles saves money.

\section{Project Importance}

Currently, efforts in WDM technology are focused on advancing the transmitter side. The attention given to the receiving element is far less than its more glamorous laser counterpart, yet advances in receiver technology could lower the high tolerances required for lasing operations in the WDM environment. WDM receivers have applications in the long-haul environment in repeater and switching technology. An array of WDM receivers could be used as a switching component for optical data packet networking. If manufacturing costs for a WDM receiver could be low enough to compete with that of the passive fiber optic component market, a cheaply made WDM receiver could become ubiquitous as a networking component. WDM receivers could be embedded components in set-top box technology to enable direct fiber to home connections.

From the software standpoint, we hope to provide tools to make the WDM technology a realizable alternative. The finite difference simulation tools could be customized in such a fashion to apply to specific fiber optic constraints of materials constants. Thus, it could be used as a standalone product in research environments to model a number of difficult problems in the fiber optics domain. 


\section{Project Background and General Methodology}

With the exponential increase in the use of the Internet, the current telecommunications infrastructure will soon be taxed to its maximum capacity - if that hasn't happened already. Multi-mode fiber and wave division multiplexing (WDM) technologies are solutions to this dilemma. Current WDM technologies involve passive components. We intend to explore the possibility of electronically controlled active components in WDM applications. The intended implementation uses microring or microdisk geometries with an active component for use as a WDM receiver.

In order for the microcavity filters to play a useful role in the telecommunications industry's 1.55 micron based technology, the pass-bands and stop bands of the filters should be compatible with channel spacing on the order of $100 \mathrm{GHz}$ and function within the $30 \mathrm{~nm}$ window of operation of erbium-doped optical amplifiers. A review of the literature shows that the initial microring resonators, based on weakly guiding material, were not really behaving as filters. Double and triple ring filters using a vernier effect where the overall FSR of the rings is comprised of overlap of the filter responses from multiple rings has been described as a method to increase the FSR. However, the FSR for the weakly guided rings is still not large enough to accommodate many channels. To increase the FSR, various researchers have adopted nanofabrication techniques and the use of strongly guided waveguides. FSR exceeding the 30-nm erbium window have been reported using microcavities of 5-micron diameter fabricated in the $\mathrm{AlGaAs} / \mathrm{GaAs}$ material system. Issues of dispersion and input/output coupling remain to be solved as the strongly guided waveguides have dimensions smaller than 1 micron. 
In order to provide low-cost WDM devices with relaxed coupling tolerances, we recently began investigating polymer materials. Though the waveguides did develop, they showed undesirable sinusoidal waviness in addition to the desired linear geometry along portions of their structure. If using the larger scale polymer structures can replicate the filter characteristics of the strongly guided materials, an opportunity for low-cost WDM devices exists. However, if the lower indices of refraction and index contrasts available in the polymer system do not allow such devices, we may be able to combine the polymers with the semiconductor systems to gain both the filter advantages of the semiconductors and the coupling ease of the larger polymer structures. Once the recipes for waveguide production are realized, we will add the microring or microdisk resonators mindful of the bending losses possible in low index contrast materials.

Dr. Axel Scherer of the California Institute of Technology has constructed arrays of microrings using nanofabrication techniques. One problem with this method is that device characteristics are unknown until the actual physical devices are created. Attempts to model the devices with computers have proved problematic. Twodimensional modeling is quite simple, but the two-dimensional simulations do not conform well to the actual behavior of the physical implementation in three dimensions. Three-dimensional modeling is beyond the physical capabilities of most computers using the current finite difference time domain methods.

We have obtained the California Institute of Technology's FFDTD package [2] from Axel Scherer in order to calculate the electromagnetic fields and visualize the field data. The FFDTD package uses Finite Difference Time Domain numerical methods and additionally uses Fourier Filters to extract single frequency modes. The libraries utilize 
many other software packages, and the different parts are quite modular. It is composed of the following:

- Structure Generation Library

- Field Generation Library

- VTK Plotting Library

- FFDTD Solver

In order to solve a problem, initial electromagnetic fields and structure geometry must be created. Electric and magnetic fields are created using the Field Generation library, while structure geometry is created using the Structure Generation library. Figure 1 shows a sample image of a structure in its initial electric field. A number of additional options exist for dielectric constant, boundary condition, and sigma variability. The Structure and Field Generation libraries generate two types of data files. The first type is actually used by the solver routines to generate the field data. The second type is a data structure used by the Visualization Toolkit [3], or VTK, to render the data into a viewable image. Both files are ASCII text files.

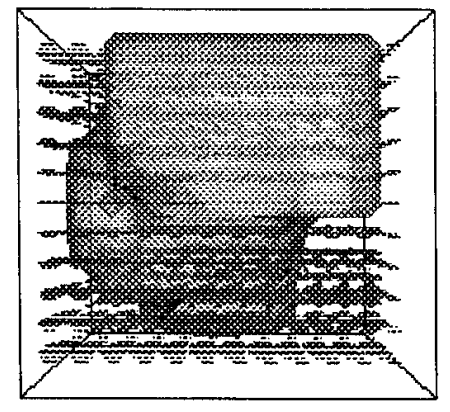

Initial Field

Vector Plot

Figure 1 


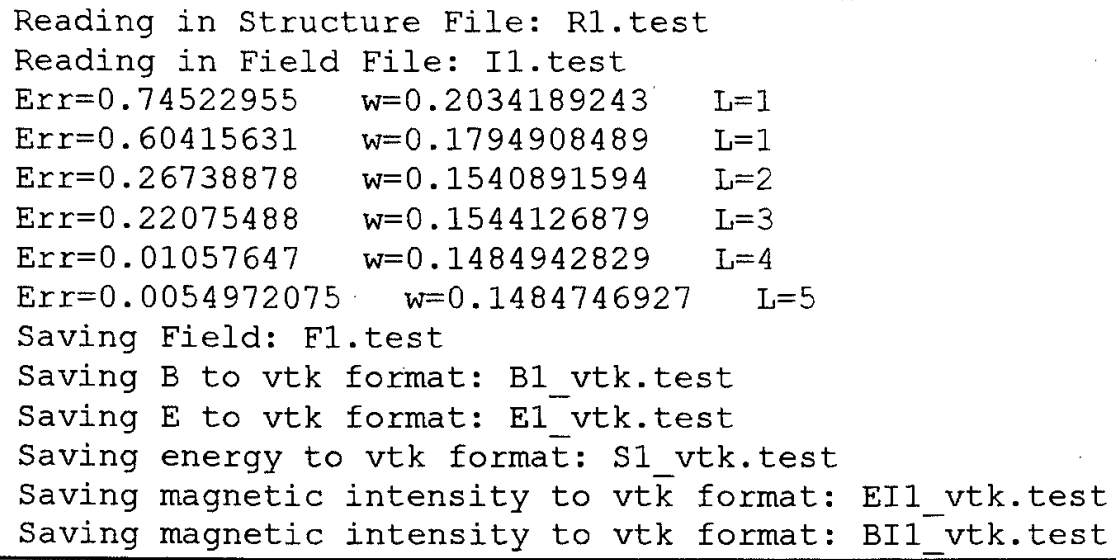

\section{Figure 2}

Once the initial conditions are created, the datasets are fed into an iterative solver routine (Figure 2). The FFDTD solver also outputs two types of files: modified electric and magnetic field datasets for VTK visualization, as well as field datasets for subsequent runs of the FFDTD solver. Finally, the VTK plotting library uses VTK function calls to visualize data. VTK is an open source, freely available software system for 3D computer graphics, image processing, and visualization. The VTK plotting library allows one to visualize data as vector plots, intensity image maps, or stream plots. Three sample images of each of the plots are shown in Figure 3, Figure 4, and Figure 5 respectively. Note if these images were viewed in a VTK render window, then the user could manipulate the image in different ways via rotating, resizing, etc. Once the image is rendered, the image may be saved into either PPG or TIFF file formats. 


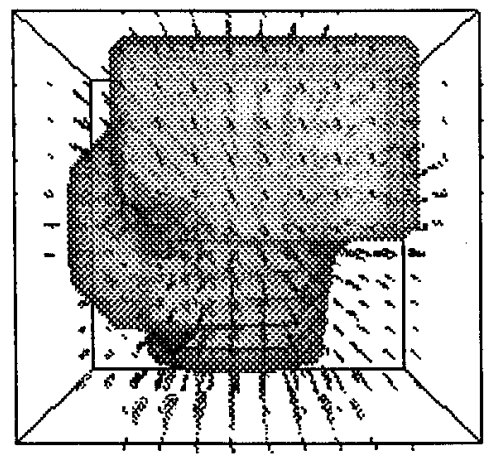

Vector Plot

Using hedgehogs

Figure 3

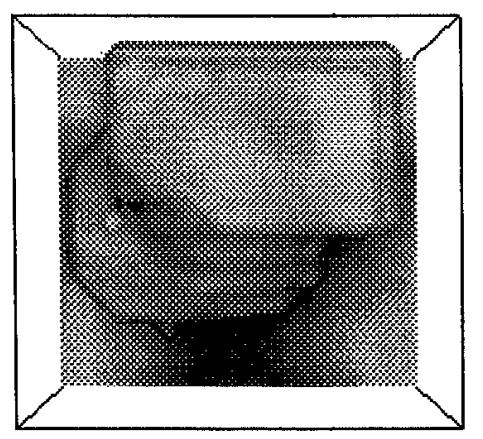

Intensity Image

Over Z-axis slice

Figure 4 


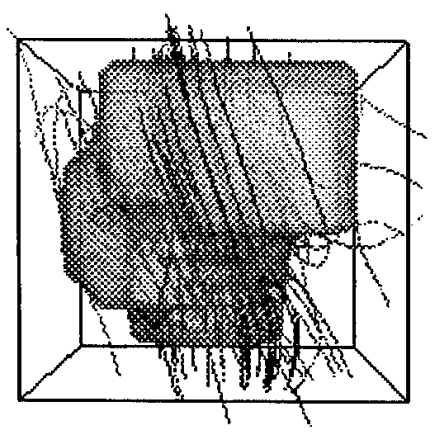

\section{Stream Plot}

Figure 5

The FFDTD solver routines face the issue that the initial boundary grid is usually of an insufficient size to accurately model a structure. When the grid size is small, the fields interact with the boundaries and produce results that do not correspond to real phenomena. The grid size is purposely kept small due to the physical memory and processing limits of the machine where the software is running. James Cole's numerical code should allow an order of magnitude increase in grid size with the same accuracy while only sacrificing a linear increase in computation time. The problem scope is still very large, so in order to achieve results within an appreciable time scale, attempts have been made to port the source code to the Tera supercomputer.

For ease of execution, the FFDTD libraries were composed of TCL scripts that directly call the $\mathrm{C}$ library functions. TCL allows a direct one-to-one mapping of $\mathrm{C}$ functions by using code wrappers. Unfortunately, the Tera does not currently possess the TCL package. Thus, short programs were written in $\mathrm{C}$ to directly call the functions. These short $\mathrm{C}$ programs were run on the Tera to generate the same ASCII text output files 
that the FFDTD solver generated. In order to verify the accuracy of the results obtained from the Tera, the algorithms were also ported to the Linux PC platform. The initial field and structure generation and the visualization aspects can still take place on platforms that support VTK and the FFDTD library in full, as these areas are not computationally bound.

As expected, many difficulties were encountered using the Tera remotely from Colorado. Most resulted from the fact that the operating system support on the Tera is still quite new. The worst of these problems manifested itself in simple file $\mathrm{I} / \mathrm{O}$ operations where the ASCII text data files were read and written. Dr. Harry Jordan devised an innovative solution to maximize the hardware benefits of the Tera's hardware multi-threaded architecture [4]. Other hardware and software problems were a disk controller failure that resulted in lost days and $\mathrm{C}$ compiler bugs.

Once a number of images are available at different time deltas, they may be strung together to form a movie. Mpeg_encode [5], a freely available, mpeg encoder from the University of California at Berkeley, is available on the Linux PC platform to encode VTK PPG stills into MPEG1 movie format. An example movie using images created from the FFDTD library follows. 


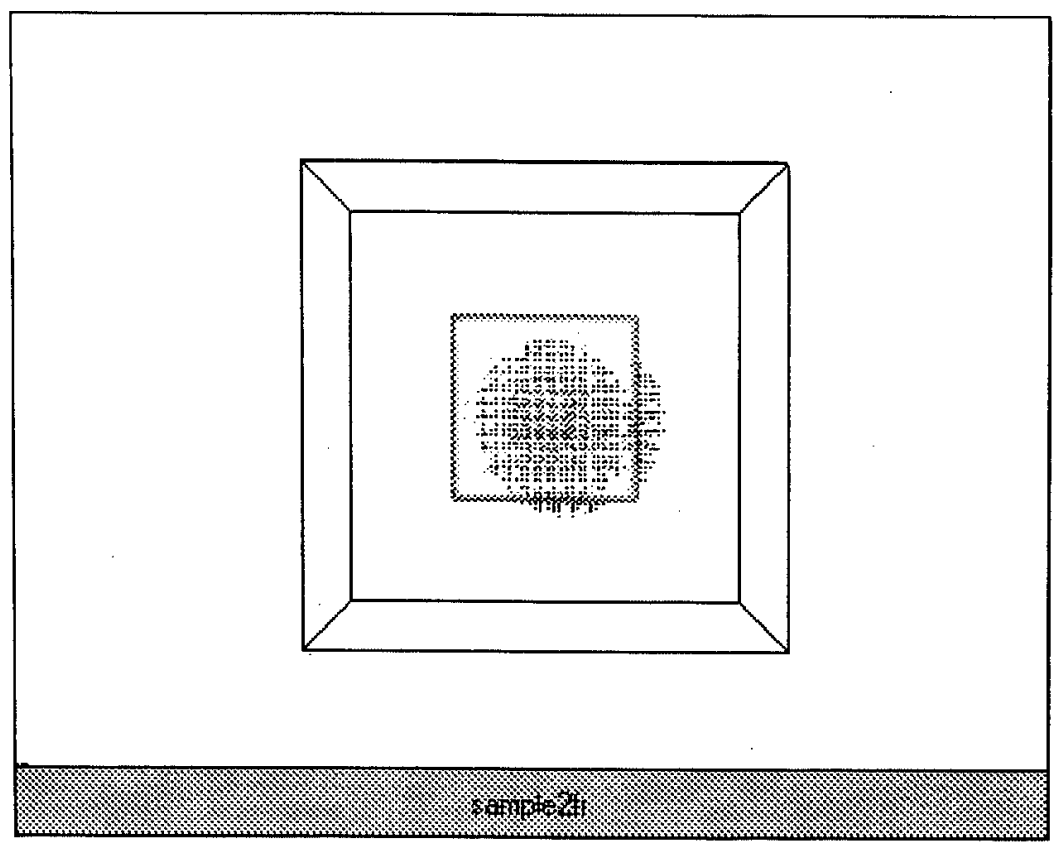

Figure 6

\section{Project State after Completion of Phase I}

At present, the scientific portions of the computer simulation tools have still yet to be completed. James Cole's numerical methods are still being ported and verified for accuracy on the Tera Supercomputer. Materials and dielectric constants must be defined for the polymers being used in the fabrication processes.

The overall infrastructure for visualization is in place. The FFDTD VTK visualization libraries can be used in conjunction with the MPEG encoder to generate MPEG 1 movies. Still images are rendered using the VTK libraries, and these images are saved into the PPG file format, which is understood by the MPEG encoder.

\section{Intellectual Property, Marketing Efforts and Business/Commercialization Strategy}

All involved parties have permitted the software distribution. James Cole's algorithms are published, and the CalTech FFDTD code has a public use license. The 
commercial prospects of a software simulation tool are quite limited as the current software is not yet up to production quality standards. Its use in a research environment is not overlooked, however. Furthermore, the possibility of developing a real application for use on the Tera supercomputer is an attractive accomplishment.

Regarding commercial opportunities, Val Morizov of E-Tek Dynamics has proposed an optical switch model using WDM components. Possibilities exist for a sample display with that technology at future trade shows. A meeting with numerous Level3 employees was set up in October, 1999, to discuss possible optical technologies. Results are pending with their research arm. Additional presentations have been made to the venture capital group University Ventures, also with results pending.

\section{Conclusions}

This project is pushing the development of new software application development on different platforms. We are attempting to utilize new numerical methods in finite difference application development. We are also attempting to develop these methods on a new supercomputer platform, the Tera. As a side effect of developing a new application for the Tera, we are also helping with the debugging of the application development environment. To this end, we have uncovered some $\mathrm{C}$ compiler bugs, and Dr. Harry Jordan has developed new file J/O procedures to maximize the Tera's capabilities with certain specific input streams. His work may be published in the future. One must not lose sight of the fact that these computer developments will pave the way towards new optical technologies that will revolutionize the telecommunications market. 


\section{References}

[1] Cole, James. IEEE-Trans. On Microwave Theory and Techniques, " $A$ HighAccuracy Realization of the Yee Algorithm Using Non-Standard Finite Differences," June 1997, vol. 45, no. 6, pp. 991-996.

[2] D’Urso, Brian. FFDTD. California Institute of Technology, 1998.

[3] Visualization Toolkit. http://www.kitware.com/vtk.html

[4] Jordan, Harry. "Parallel Formatted I/O in Shared Memory Multiprocessors."

[5] Rowe, Lawrence A., Kevin Gong, Eugene Hung, Ketan Patel, Steve Smoot, and Dan Wallach. MPEG-1 Video Software Encoder. University of California at Berkeley, Computer Science Division-ECCS, 1995. 